\title{
A Hybrid COPY-MOVE Forgery DETECTION TECHNIQUE USING REgIONAL SIMILARITY INDICES
}

\author{
Rani Susan Oommen ${ }^{1}$ and Dr. Jayamohan $\mathrm{M}^{2}$ \\ ${ }^{1}$ Sree Buddha College of Engineering for Women, Kerala, India \\ ${ }^{2}$ College of Applied Science, Adoor, India
}

\begin{abstract}
Different methods have been experimented for processing and detecting forgery in digital images. Image forgery involves various activities like copy-move forgery, image slicing, retouching, morphing etc. In copy-move forgery a portion within the image is copied and pasted on another part of the same image, generally to conceal or enhance certain portions of the image. This paper proposes a copy-move forgery detection using local fractal dimension and structural similarity indices. The image is classified into different texture regions based on the local fractal dimension. Forgery checking is thus confined to be among the portions within a region. Structural similarity index measure is applied to each block pair in each region to localize the forged portion. Experimental results prove that this hybrid method can effectively detect such kind of image tampering with minimum false positives.
\end{abstract}

\section{KEYWORDS}

Copy-move forgery detection, image forensics, local fractal dimension \& structural similarity index.

\section{INTRODUCTION}

The secure exchange and storage of authentic data in digital format has become the biggest concern in the era of paperless workplaces and electronic services. Though all types of files, like documents, voice data, and image can be well manipulated, images are more vulnerable mainly due to the limitations of human visual system. Rapid advancement in imaging technology and availability of powerful digital image processing programs such as Photoshop, GIMP etc., makes it relatively easy to manipulate digital images. Therefore establishing the authenticity of an image has become a challenging task.

Image forgery can be defined as the intentional manipulation of images for purposes which can be malicious ornon-malicious. The act of presenting forged images can be traced back to as early as 1840s. The self Portrait of a Drowned Man, created by Hippolyte Bayrad is considered to be the very first fake image, in which he was shown committing suicide [1]. Many such incidents of image forgery have been reported since then. Digital image forensics is a field that analyses images of a particular scenario to establish the credibility and authenticity through a variety of means. Digital image forgery detection techniques are generally categorized into active approach and passive approach. In active approaches such as digital watermarking and digital signature, watermarks must be embedded during image acquisition. In this type of detection, access to original image is required to insert a watermark which is practically infeasible [2]. In passive approach, no prior information is required. 
The common image tampering techniques include: Copy- move forgery, Image splicing, Image retouching, Morphing and Image Enhancement. Copy-move forgery is the most common image tampering technique which copies a region (source region) in the image and then paste the region to another part of the same image (target region), in this way to deliberately conceal the undesirable objects of the original image [3] [4]. The main objective of copy-move forgery detection methods is the verification of the trust worthiness of the digital image, by detecting the particular type of forgery and locating the forged portion, if possible.

During the past few years, there have been a lot of attempts reported in copy move forgery detection. The simplest method for detecting copy move is by exhaustive search, where an image and its circularly shifted version are overlaid looking for closely matching image segments [5].Another method is based on auto correlation properties. This approach is shown to be effective only when the forged regions were a large portion of the image. The copy-move forgery detection techniques can be either block-based, key-point based or hybrid approaches. In block-based approach, the image is segmented into overlapping blocks first. The task here is to detect connected image blocks that are copied and moved, instead of detecting the whole duplicated region. Fridich et.al, suggested the first exhaustive search block-based method to detect copy move forgery based on DCT coefficients [5]. This method can detect copy move region, but it will not working noisy image. Popescu and Farid presents a dimensionality reduction based copymove forgery detection by applying PCA to blocks in the image, which is robust to additive noise or lossy compression [6]. Another block-based approach proposed by Luo et.al detects copymove forgery based on blockcharacteristicsfeaturesincludingthedirectionalinformationofblocks. It has lower computational complexity and robust to post-copying manipulations such as noise contamination, blurring, mixture of these operations etc.[7]. A block based approach which exploits texture as features to detect copy move forgery was proposed by Ardizzone et.al. [8]. The algorithm uses five texture descriptors, namely, statistical descriptors (mean, standard deviation, skewness and kurtosis), edge histogram, Tamura descriptors (contrast, coarseness and directionality properties from Tamura setoff features), Gabor Descriptors and Haralick descriptors. Blocks are sorted and similarity criterion is applied to find can did ate blocks. X.Quan, H.Zhang proposed copy move detection method confined to image blocks with similar texture, instead of in the whole image, which decreases the complexity of the algorithm [9].

In this paper, a hybrid passive approach to copy-move forgery detection is proposed. Given a forged image, local fractal dimension (LFD) based classification phase followed by block matching phase based on structural similarity index measure (SSIM) is used to identify suspected copy-move forged region. The following section of the paper is organized as follows: Section 3 describes the proposed hybrid method along with its theory. The results and analysis is given in Section 5 and section 6 concludes the paper and future work is discussed.

\section{Proposed SCHEME}

The aim of the proposed work is to develop an improved method for copy-move forgery detection by using local fractal dimension for image segmentation and estimating SSIM of block pair in each segmented region to localize the forged region. The framework within which the algorithm proceeds is shown in Fig1. Main objectives of the work are:

- Identify the portion of the image where copy-move forgery has been done.

- Use local fractal dimension for efficient texture segmentation of image.

- To develop detection method that is robust to blurring, noise contamination and compression.

This work develops in two phases. Firstly, the image is classified based on local fractal dimension, estimated for each block. In the second phase, localize the forged region in that image. 
The given suspected image is converted into gray scale. Image is divided into overlapping blocks of fixed size(e.g. $32 * 32)$.Estimate the local fractal dimension of each block using differential box counting method $(\mathrm{DBC})$. This estimated local fractal dimension is used as feature vector for image classification (texture based) [10]. Since the copied and moved region has similar texture, segmenting the image into different texture regions will reduce the no: of comparisons between blocks. The feature vector in each segment is then lexico graphically sorted. Therefore first phase results indifferent texture regions of image which form the input to the second phase. In the second phase, estimate the SSIM (Structural Similarity Index measure)of each block pair in each region. On comparison of the SSIMs between each block pair, we can identify potential duplicated blocks in the image. Perform matching and localization of forged regions.

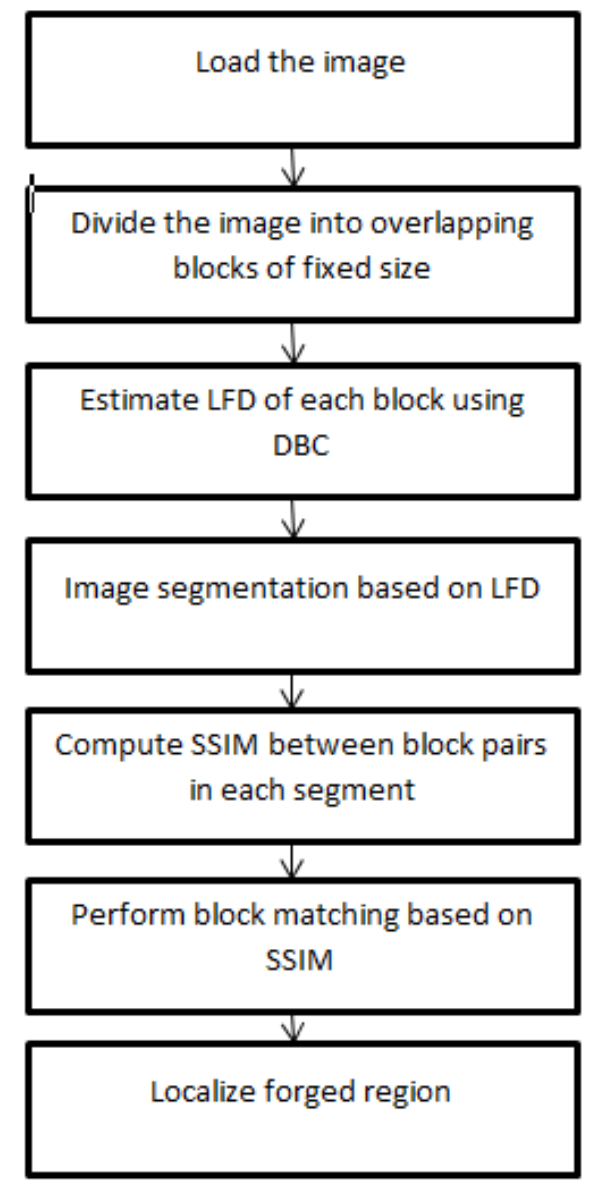

Figure 1. Framework of the proposed work

\subsection{Basic Procedure}

The procedure to detect copy-move forgery can be summarized as follows:

1. Suspected image is read as pixel intensity values.

2. Divide the image into overlapping blocks of equal size (taken as square sized blocks, with block size in powers of 2 , for computational convenience).

3. Estimate local fractal dimension (LFD)of each block using differential box counting method (DBC). 
4. Image blocks are separated into different groups based on LFD values.

5. Blocks within each group are checked for forgery.

5.1 Estimate structural similarity index of each pair of blocks.

5.2 Blocks with SSIM values higher than 0.8 are suspected for forgery. The threshold is selected considering the possible distortions within the image due to transmission errors, lossy compression etc.

6. Localization of the forged region.

\subsection{Fractal Dimension}

According to the notions of Euclid geometry, an objector a scene can have only integer dimensions. The the oryof fractal geometry presents a different observation. It is difficult for a natural scene to have integer dimension, instead they are having fractional dimensions. The fractal dimension of an object will be always less than its conventional dimension[11]. Barnsley presents fractal dimension (FD) as a quantity that can measure the similarity of two fractals Fractal dimension estimates how densely a fractal occupies the metric space in which it lies[12].

The most basic method to determine fractal dimension is box-counting theorem. Let $\mathrm{A}$ be the matrix representing the image. Let the space $\mathrm{R}^{2}$ be covered by closed square boxes of side length $1 / 2^{\mathrm{n}}$. Let $\mathrm{N}(\mathrm{A})$ denote the number of boxes which intersect $\mathrm{A}$. Then the box counting theorem states that, the fractal dimension D of A can be obtained as

$$
D=\lim _{n \rightarrow \infty} \frac{\log (N(A))}{\log \left(2^{n}\right)}
$$

For an object with deterministic self-similarity, its FD is equal to its box-counting dimension. However, natural scenes are not the ideal deterministic fractals. The basic box counting method, since it requires the image in black and white, is not well-suited for estimating FD of gray scale images. With Differential Box Counting method, Sarkar and Choudhari [10]proposed assign if I cant improvement over box counting which makes it better suited for estimating complexity of two dimensional gray scale images.

In Differential Box counting (DBC), an image is considered as a three-dimensional spatial surface with $(\mathrm{x}, \mathrm{y})$,denotes the pixel position on the image plane, and the third coordinate(z) denoting pixel gray level. Consider an image of size $\mathrm{M} * \mathrm{M}$. The $(\mathrm{x}, \mathrm{y})$ plane is partition edintononoverlappingblocksofsize,say,s*s. Thescaleofeachblockisr $=\mathrm{s}$, where $\mathrm{M} / 2 \geq \mathrm{s}>1$ ands is an integer. One ach block there is a column of boxes of size $\mathrm{s} * \mathrm{~s} * \mathrm{~s}^{\prime}$ wheres' is the height of each box, $\mathrm{G} / \mathrm{s}^{\prime}=\mathrm{M} / \mathrm{sand} \mathrm{G}$ is the total number of gray levels. Let the minimum and maximum gray level in the (i,j)-th block fall into the k-th and l-th boxes, respectively. The boxes covering this block are counted in the number a $\operatorname{sn}_{\mathbf{r}}(\mathrm{i}, \mathrm{j})=1_{1-k+1}$, wherethesubscriptrdenotestheresultusingthescaler.Consideringcontributions formal 1 blocks, then the number of boxes $\mathrm{N}_{\mathbf{r}}$ is counted for different values of $\mathrm{r}$ as:

$$
N_{r}=\sum_{i, j} n_{r}(\mathrm{i}, \mathrm{j})
$$

Then the FD can be estimated from the least squares linear fito flog $\left(\mathrm{N}_{\mathbf{r}}\right)^{\mathrm{versus}} \log (1 / \mathbf{r})$.

Conci and Campos has published an improvement over DBC[13]. The image division in boxes of different length is processed in a different manner from basic DBC. The image is divided into boxes of size nxnxn' forn $=2,4,8 \ldots 2$ mandn' $=2,4,8 \ldots 2$ m' foreachimage subdivision, where n' is the height of the box. Then the number of boxes $\mathrm{N}$ is counted as: 
International Journal of Computer Science \& Information Technology (IJCSIT) Vol 7, No 4, August 2015

$$
\mathrm{N}=\sum \mathrm{n}(\mathbf{i}, \mathbf{j})
$$

where, n=int $(\operatorname{Graymax}(\mathrm{i}, \mathrm{j}) / \mathrm{n} \operatorname{int}(\operatorname{Graymin}(\mathrm{i}, \mathrm{j}) / \mathrm{n}$ ') +1 ,

Two smaller matrices, Graymax and Graymin are used instead of the bitmap of $M * M$ pixels in the image used to compute the fractal dimension in original DBC method. This modification improves the computational speed of DBC with simplifying the operation.

In this method, we use the differential-box counting method proposed by Sarkar and Choudhari [10] to calculate fractal dimension. The value of FD will be stable over a pure fractal image. But natural images are not having pure fractal nature. Hence if we estimate fractal dimensions of local areas of the image, it will be different from the FD of the entire image and the results may vary depending on the local texture variations. Textured regions are used as ideal parts for copy- move forgery, since texture dare as have similar colour and noise variation properties to that of the image which are un perceivable for human eye looking for in consistencies in image statistical properties. Local fractal dimension [14] [15] can be used to identify variations in images. Hence it can be used as a measure for texture similarity for texture based classification.

\subsection{Structural Similarity Index Measure (SSIM)}

In order to perform block matching, Structural Similarity Index Measure (SSIM) of each pair of blocks is computed within each region. The structural similarity (SSIM) metric and its multi-scale extension (MS-SSIM) is a method for measuring similarity between two images[16] [17].Natural images signals' are highly structured, the irpixels exhibit strong dependencies, especially when they are spatially proximate, and these dependencies carry important information about the structure of the objects in the visual scene. The structural similarity (SSIM) metric and its multiscale extension (MS- SSIM) evaluate visual quality based on the premise that the human visual system (HVS) has evolved to process structural information from natural images and hence, a high- quality image is one whose structure closely matches that of the original. SSIM employs a modified measure of spatial correlation between the pixels of the reference and test image segment to quantify the degradation of an images structure. MS-SSIM extends SSIM through a multi-scale evaluation of this modified spatial correlation measure.

SSIM quantifies visual quality with a similarity measure between two patches $\mathrm{x}$ and $\mathrm{y}$ as the product of three components: mean $\mathrm{m}(\mathrm{x}, \mathrm{y})$, variance $\mathrm{v}(\mathrm{x}, \mathrm{y})$, and cross- correlation $\mathrm{r}(\mathrm{x}, \mathrm{y})$. The two patches $\mathrm{x}$ and $\mathrm{y}$ correspond to the same spatial window of the image $\mathrm{X}$ and Yrespectively. The system separates the task of similarity measurement into three comparisons: luminance, contrast and structure.

The local statistics are computed within a local $8 * 8$ square window, which moves pixel-by-pixel over the image region. At each step, the local statistics and SSIM index are calculated within the local window. One problem with this method is that the resulting SSIM index map often exhibits undesirable "blocking" artifacts. Let ' $w$ ' be symmetric Gaussian weighting function $w=\left\{w_{i}, \mathrm{i}=\right.$ $1,2 \ldots . \mathrm{N})$. The Local statistics are then modified as:

$$
\begin{aligned}
& \mu_{x}=\sum_{1=1}^{N} x_{i} w_{i} \\
& \sigma_{x}=\left(\sum_{i=1}^{N} w_{i}\left(x_{i}-\mu_{x}\right)^{2}\right)^{1 / 2} \\
& \sigma_{x y}=\sum_{i=1}^{N} w_{i}\left(x_{i}-\mu_{x}\right)\left(y_{i}-\mu_{y}\right)
\end{aligned}
$$


With such a windowing approach, the quality maps exhibit a locally isotropic property. In practice, a single overall quality measure of the entire image region is calculated using mean SSIM (MSSIM) index:

$$
\operatorname{MSSIM}(\mathrm{X}, \mathrm{Y})=\left(\sum_{j=1}^{M} \operatorname{SSIM}\left(x_{i}, y_{j}\right)\right) / \mathrm{M}
$$

where, $\mathrm{X}, \mathrm{Y}$ are the two comparing image regions respectively, $x_{i}$ and $y_{j}$ are the image contents at the $\mathrm{j}$-th local window and $\mathrm{M}$ is the number of local windows in the image region. The resultant SSIM index is a decimal value between -1 and 1 , and value 1 is only reachable in the case of two identical sets of data.

\section{EXPERIMENTAL RESULTS}

The method has been tested with a collection of images of different types. Copy-move forgery was performed on the images to hide certain features in the original image. After duplicating a region within the image different types of editing operations like noise addition was performed on the image. The method has been tested on images after jpeg compression also. Considering the fact that natural images may exhibit similar regions even without forgery, the proposed algorithm proceeds based on the assumption that duplicated region less than $1 \%$ of the whole image is not suspected forged. The algorithm is coded in MATLAB 2011a on a machine equipped with i3 $2.4 \mathrm{GHz}$ processor with $4 \mathrm{~GB}$ RAM. The original non-forged image used is in Fig. 2a and Fig. 3a. After performing copy-move forgery, Fig. $2 b$ and Fig. $3 b$ is used as test image for experimentation. The phase 1 output, after classification into different texture regions is shown in Fig. 2c and Fig. 3c. Finally, after phase 2, those regions that have similar texture and quality are suspected as duplicated regions and are highlighted in Fig. 2d and Fig. 3d.

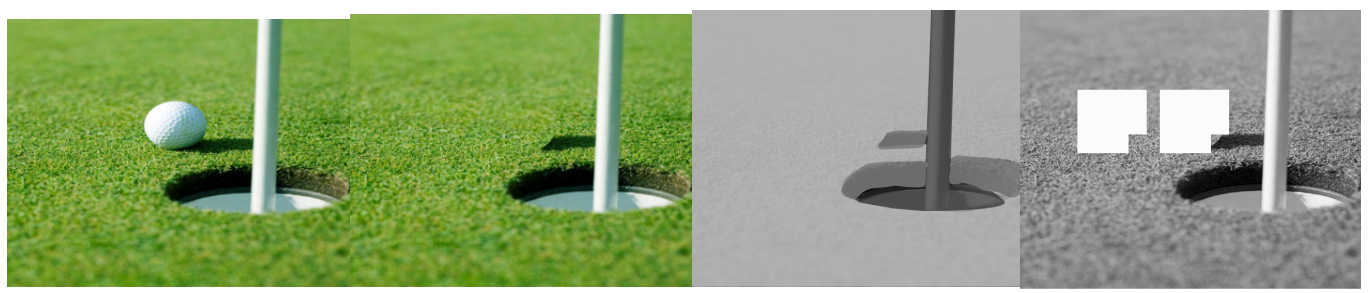

(a)

(b)

(c)

(d)

Figure 2. Example image set 1

In order to define the success rate of the proposed algorithm on the test samples of hundred images, we define the parameter, correct detection rate $(\gamma)$. The image is tested with varying block size, $8 * 8,16 * 16$ and $32 * 32$. In each case the correct detection rate against post processing operations such as JPEG compression, blurring and noise addition is shown in Table 1. The experimental result shows the efficiency and of the proposed method. It also indicates the robustness of the proposed detection scheme to JPEG compression, blurring and noise addition.

Table 1. Correct Detection rate on Test Image.

\begin{tabular}{|l|l|l|l|}
\hline \multicolumn{1}{|c|}{$\gamma$} & \multicolumn{1}{|c|}{$8 * 8$} & \multicolumn{1}{c|}{$16 * 16$} & \multicolumn{1}{c|}{$32 * 32$} \\
\hline Unprocessed image & 0.9981 & 0.9965 & 0.9952 \\
\hline JPEG compression & 0.9978 & 0.9942 & 0.9931 \\
\hline Blurring & 0.9961 & 0.9937 & 0.9929 \\
\hline Noise & 0.9975 & 0.9954 & 0.9941 \\
\hline
\end{tabular}




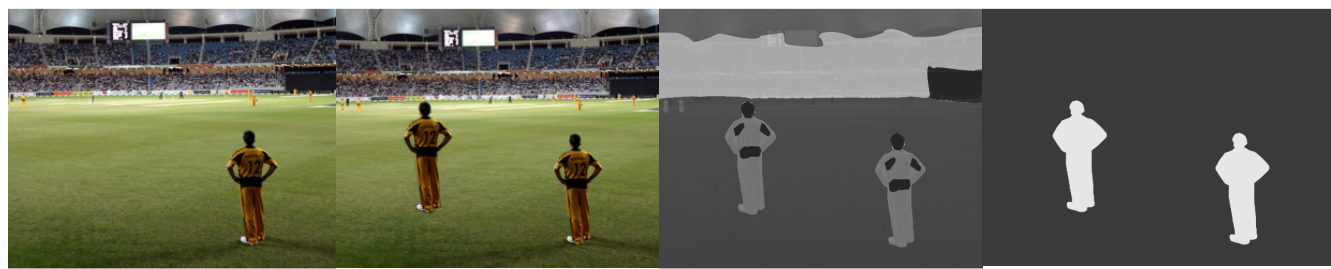

(a)

(b)

(c)

(d)

Figure 3. Example image set

\section{CONCLuSion}

In this work, a for ensic algorithm to localize copy-move forged regions in a digital image without any a priori knowledge about the location of the possibly tampered areas has been presented. In this work a hybrid approaches issued to localize the manipulated areas. Measurement of local fractal dimension issued to classify image into different texture regions. The concept of structural similarity index measure issued to extract the manipulated area. The experimental results hows that the proposed method can be avalid toolf or detecting and localizing copy-move forgery in digital images. The inherent advantage of SSIM helps to detect forgery even when duplicated image is noise contaminated or blurred etc. The major drawback of fractal coding systems is the high encoding time requirements. Future work of this method can be to use a different measure instead of SSIM to localize forged regions. Since SSIM is not a good measure to compare regions that are subjected to post-processing region duplication such as scaling, rotation etc., an alternate estimate can be experimented instead of SSIM to localize forged regions. Use of hybrid measures can also be attempted along with fractal dimension can be experimented as the future work to efficiently detect copy-move forgery.

\section{REFERENCES}

[1] J. A. Redi, W. Taktak and J. L Dugelay, Digital Image Forensics: A booklet for beginners, Multimedia Tool Appl., vol 51, no:1, pp.133-62, Jan. 2011.

[2] J. Fridich, Methods For Tamper Detection In digital Image, Proc ACM Workshop onMultimedia and Security, Orlando,, FL, October 30-31, 1999, pp. 19-23.

[3] Qazi, Tanzeela, et al. "Survey on blind image forgery detection." Image Processing, IET 7.7 (2013): 660-670.

[4] Oommen, Rani Susan, M. Jayamohan, and S. Sruthy. "A Survey of Copy-Move Forgery Detection Techniques for Digital Images."

[5] J. Fridich, D Soukal and J Lukas, Detection of Copy- Move Forgery in digital images,Proc of Digital Forensic Research Workshop, Aug, 2003.

[6] A. C Popescu, H. Farid, Exposing digital forgeries by detecting duplicated regions, Technical report TR 2004-515, Dartmough college, Aug2004.

[7] W. Luo, J Huang and G. Qio, Robust detection of region-duplication forgery in digitalimage, Proc of ICPR, Aug 2006.

[8] E. Ardizzone, A. Bruno, and G. Mazzola, Copy-move forgery detection via texturedescription, in Proceedings of the 2nd ACM workshop on Multimedia in Forensics,Security and Intelligence (MiFor 10), pp. 5964, ACM, October 2010.

[9] X. Quan, H. Zhang, Copy-move Forgery detection in Digital Images Based On LocalDimension Estimation, June 2013.

[10] Sarkar, N. ; Choudhuri, B.B. (1994): An efficient differential box counting approach to compute fractal dimension of image. IEEE transactions. On Syst. Man Cybernetics,24, pp.115, 120.

[11] Fisher, Y. (1995). Fractal Image Compression-Theory and Application. Springer-Verlag, New York.

[12] Barnsley, M. (1993). Fractals Everywhere, 2nd edn. Academic Press, Cambridge. 
International Journal of Computer Science \& Information Technology (IJCSIT) Vol 7, No 4, August 2015

[13] Conci, A.; Campos, C.F.J. (1996): An efficient Box-counting fractal dimension approach for experimental Image Variation Characterization. Proceedings of IWISP96- 3rd InternationalWorkshop in Signal and Image Processing, Elsevier Science, UK, pp.665-668.

[14] Jayamohan, M., and K. Revathy. "An Improved Domain Classification Scheme Based on Local Fractal Dimension." Indian Journal of Computer Science and Engineering (IJCSE) 3.1 (2012): 138145.

[15] Backes, André Ricardo, and Odemir Martinez Bruno. "Shape classification using complex network and multi-scale fractal dimension." Pattern Recognition Letters 31.1 (2010): 44-51.

[16] Wang, Zhou, et al. "Image quality assessment: from error visibility to structural similarity."Image Processing, IEEE Transactions on 13.4 (2004): 600-612.

[17] Rouse, David M., and Sheila S. Hemami. "Understanding and simplifying the structural similarity metric.” Image Processing, 2008. ICIP 2008. 15th IEEE InternationalConference on. IEEE, 2008. 